\title{
A database of 389 medicinal plants for diabetes
}

\author{
Padavala Ajay Babu ${ }^{*}$, Gadde Suneetha ${ }^{1}$, Radha Boddepalli ${ }^{1}$, Vedurupaka Vasantha Lakshmi ${ }^{1}$, Talluru Sudha Rani ${ }^{1}$, Yellapu \\ RamBabu $^{2}$ and Kolli Srinivas ${ }^{3}$ \\ ${ }^{1}$ Bioinformatics Training \& Research Centre, ProGene Biosciences, 103, Bharat Towers, Dwaraka Nagar, Visakhapatnam - 530016; \\ ${ }^{2}$ Department of Biotechnology, College of Engineering, GITAM, Visakhapatnam - 530045; ${ }^{3}$ Sri Venkateswara College of Pharmacy, \\ Etcherla, Srikakulam; \\ Padavala Ajay Babu* - Email: ajay@progenebio.in; Phone: +91 891 5571195; * Correspondence author \\ received March 17, 2006; accepted April 5, 2006; published online April 13, 2006
}

\begin{abstract}
:
Medicinal plants used to treat hypoglycemic and hyperglycemic conditions are of considerable interest to ethno-botanical community as they are recognized to contain valuable medicinal properties in different parts of the plant. The active principles of many plant species with desired properties are isolated to cure ailments such as diabetes type-1 and type-2, respectively. Here, we describe DiaMedBase, a database containing information of medicinal plants for diabetes.
\end{abstract}

Availability: http://www.progenebio.in/DMP/DMP.htm

Keywords: medicinal plants; diabetes; database; data; literature

\section{Background:}

Since olden days, plants are used to treat many ailments. India has about 45,000 plant species and several thousands have been claimed to possess medicinal properties. [1] Medicinal plants used to treat hypoglycemic or hyperglycemic conditions are of considerable interest for ethno-botanical community as they are recognized to contain valuable medicinal properties in different parts of the plant and a number of plants have shown varying degree of hypoglycemic and anti-hyperglycemic activity. [1] The active principles of many plant species are isolated for direct use as drugs, lead compounds or pharmacological agents. [3] Several species of medicinal plants are used in the treatment of diabetes mellitus, a disease affecting large number of people world-wide. Traditional plant medicines or herbal formulations might offer a natural key to unlock diabetic complications. [2]

Diabetes mellitus is the major endocrine disorder [4] responsible for renal failure, blindness or diabetic cataract [5], poor metabolic control [6], increased risk of cardiovascular disease including atherosclerosis and AGE (advanced glycation end) products. [7] Antioxidants play an important role to protect against damage by reactive oxygen species and their role in diabetes has been evaluated. Many plant extracts and products were shown to possess significant antioxidant activity. [8] Hence, we created DiaMedBase, a diabetes literature database of medicinal plants with abstract, plant parts, objective and a 'disease link' to diseases other than diabetes for each medicinal plant.

\section{Methodology:}

\section{Construction of DiaMedBase}

DiaMedBase is constructed using html and can be accessed at http://www.progenebio.in/DMP/DMP.htm. Data were collected from various literature sources such as, PubMed [9], ScienceDirect [10], Mary Ann Liebert [11], BlackWell Synergy [12], IngentaConnect [13], Scirus [14], Bentham Publishers [15], Wiley journals [16] and others. DiaMedBase includes 742 records, containing about 309 genus and 389 species of plants described to possess medicinal properties against diabetes. The complete list can be found at http://www.progenebio.in/DMP/listz.htm. However, the list is not complete. They are provided alphabetically and the records are organized to simplify the task of finding relevant data for any plant. The database can be accessed alphabetically using genus name for information on specific plants.

\section{Features of DiaMedBase}

Each entry in DiaMedBase is provided with a unique accession number DMPXX001, where, XX represents the first letter of genus and species, respectively. Digits refer to the record number in the database. For example, the accession number of Aloe vera is DMPAv042, where, 042 represent record entry in the DiaMedBase. The Medicinal Plant row in some records display a list of medicinal plants and the representative plants are highlighted. DiaMedBase contains 30 entries for genus Trigonella, 22 entries for Momordica, 19 for Gymnema, 13 entries for Opuntia and Panax, 11 for Allium species and 10 each for Aloe, Tinospora, respectively. A screen-shot of the DiaMedBase is given in Figure 1. Of the collected data, $36 \%$ whole plant, $26 \%$ leaves, $12 \%$ seeds, $10 \%$ roots, and $4 \%$ fruits. The characteristic feature of DiaMedBase is 'Disease Link', provided in each record displays the list of diseases other than diabetes as a pop-up window indicating the importance of medicinal values of plants.

\section{Utility:}

DiaMedBase emphasizes the importance of hypoglycemic and hyperglycemic properties possessed by medicinal plants. The database finds utility to the scientific community for a quick review on the number of plants and plant parts for diabetes medicinal plant research.

\section{Future development:}

Continuous updates shall be released to include other plants of medicinal value periodically. The present access method shall be upgraded to browse records on the studied plant parts. We plan to develop provisions to search the database to identify plants of interest using keywords. 


\section{Bioinformation}

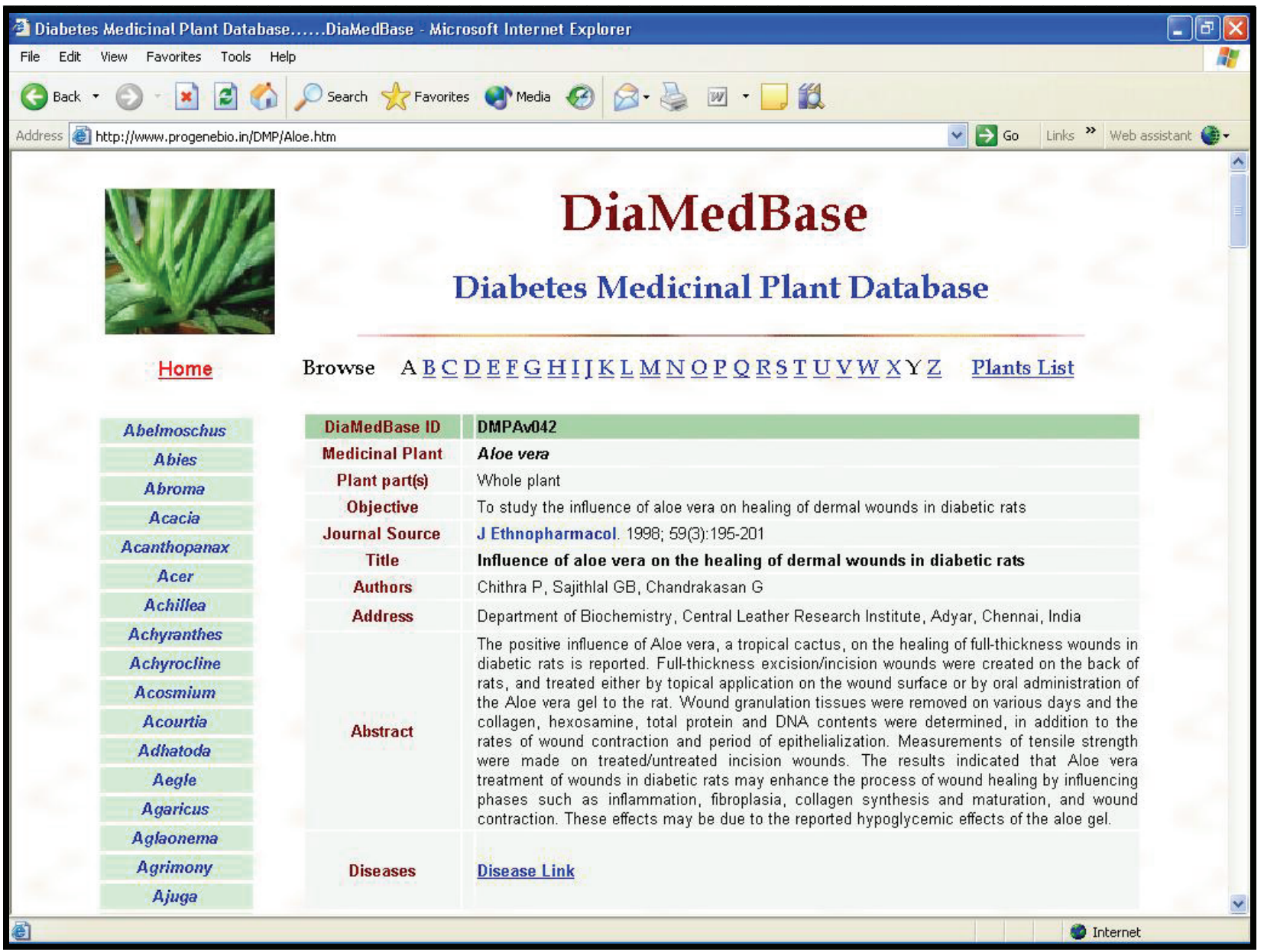

Figure 1: A screen shot of Aloe vera record entry in DiaMedBase is shown

\section{References:}

[1] J. K. Grover, et al., J Ethnopharmacol., 81:81 (2002) [PMID: 12020931]

[2] S. Nammi, et al., BMC Complement Altern Med., 3:4 (2003) [PMID: 12950994]

[3] D. S. Fabricant \& N. R. Farnsworth, Environ Health Perspect., 109:69 (2001) [PMID: 11250806]

[4] J. P. Burke, et al., Diabetes Care, 26:7 (2003) [PMID: 12832302]

[5] B. Thylefors, The WHO program for the prevention of blindness. Int. J. of Ophthal., 14:211 (1990)

[6] R. Donnelly, et. al., BMJ, 320:1062 (2000) [PMID: 10764371]

[7] T. Yokozawa \& T. Nakagawa, Food and Chemical Toxicology, 42:975 (2004) [PMID: 15110107]
[8] M. C. Sabu \& R. Kuttan, J. Ethnopharmacol., 81:155 (2002) [PMID: 12065146]

[9] http://www.ncbi.nlm.nih.gov/entrez/query.fcgi?db=Pub Med

[10] http://www.sciencedirect.com

[11] http://www.liebertpub.com

[12] http://www.blackwellsynergy.com/servlet/useragent?func $=$ showHome

[13] http://www.ingentaconnect.com/content

[14] http://www.scirus.com/srsapp/

[15] http://www.bentham.org

[16] http://www3.interscience.wiley.com/cgi-bin/home

Edited by $P$. Kangueane

Citation: Babu et al., Bioinformation 1(4): 130-131 (2006)

License statement: This is an open-access article, which permits unrestricted use, distribution, and reproduction in any medium, for non-commercial purposes, provided the original author and source are credited. 